\title{
BMJ Open Meta-analysis of the prognostic value of pretreatment serum ferritin in hepatobiliary and pancreas (HBP) cancers
}

\author{
Shuwen Lin (D) , ${ }^{1}$ Yinghua Fang, ${ }^{2}$ Ye Lin, ${ }^{3}$ Zhikang Mo, ${ }^{1}$ Xiaocheng Hong, ${ }^{1}$ \\ Zhixiang Jian, ${ }^{3}$ Chenggang $\mathrm{Ji}^{1}$
}

To cite: Lin S, Fang $Y$, Lin Y, et al. Meta-analysis of the prognostic value of pretreatment serum ferritin in hepatobiliary and pancreas (HBP) cancers. BMJ Open 2021;11:e040801. doi:10.1136/ bmjopen-2020-040801

- Prepublication history and additional supplemental material for this paper is available online. To view these files, please visit the journal online (http://dx.doi org/10.1136/bmjopen-2020040801).

SWL, YHF and YL contributed equally.

Received 27 May 2020 Revised 03 March 2021 Accepted 14 March 2021

Check for updates

(c) Author(s) (or their employer(s)) 2021. Re-use permitted under CC BY-NC. No commercial re-use. See rights and permissions. Published by BMJ.

${ }^{1}$ General Surgery, Binhaiwan Central Hospital of Dongguan, Dongguan, China

${ }^{2}$ Pain, Binhaiwan Central Hospital of Dongguan, Dongguan, China ${ }^{3}$ General Surgery, Guangdong Provincial People's Hospital, Guangzhou, China

Correspondence to Dr Chenggang Ji; jcg_999@163.com

\section{ABSTRACT}

Background and objectives Studies have shown that serum ferritin (SF) has unfavourable prognostic value in hepatobiliary and pancreas (HBP) cancers. This metaanalysis aimed to comprehensively assess the prognostic role of pretreatment SF in patients with HBP cancers. Methods Eligible studies published before January 2020 were obtained through a comprehensive search in the PubMed, Web of Science, Cochrane Library and EMBASE databases. Pooled HRs and $95 \% \mathrm{Cls}$ were then employed as effect sizes.

Results Seven studies comprising 1244 patients were pooled. Elevated pretreatment SF was associated with worse overall survival (OS) (HR 1.60, 95\% Cl 1.36 to 1.88, $\mathrm{p}<0.001)$ and recurrence-free survival/progression-free survival/time to recurrence (HR $1.70,95 \% \mathrm{Cl} 1.15$ to $2.52, p=0.008)$. Significant prognostic value of elevated pretreatment SF on OS was detected in the subgroups regardless of the cancer type, race, SF cut-off value, tumour-node-metastasis stage and Newcastle-Ottawa Scale score.

Conclusion Elevated pretreatment SF was associated with worse survival outcome of patients with HBP cancers. As such, it may serve as a novel prognostic biomarker for HBP cancers.

\section{INTRODUCTION}

Hepatobiliary and pancreas (HBP) cancers include hepatocellular carcinoma (HCC), pancreatic cancer (PC) and biliary tract cancers. Biliary tract cancers range from those affecting the gall bladder, ampulla of Vater cancers as well as intrahepatic and extrahepatic cholangiocarcinomas. HBP cancers are one of the most common causes of cancer-related deaths in many countries. Currently, the morbidity and mortality rates of HBP cancers across the world are rising rapidly. ${ }^{1}$ These cancers are characterised by a poor overall survival (OS) and a high recurrence rate even when diagnosis and treatment are performed during the early stages of the disease. Unfortunately, majority of the patients are diagnosed at terminal stage of HBP cancers. ${ }^{2-7}$ Despite rapidly developing research on diagnosis and treatment of $\mathrm{HBP}$

\section{Strengths and limitations of this study}

This study is the first systematic meta-analysis based on a comprehensive literature search to assess the prognostic value of serum ferritin in patients with hepatobiliary and pancreas (HBP) cancers.

- The results were systematically synthesised to overcome the shortcoming of a small-scale study, which reported the association between serum ferritin and HBP cancers.

- Limitations of the study will be the lack of robust clinicopathological data, the inclusion of different treatment modalities, the retrospective nature of the included studies and the publication bias among included publications.

cancers, their prognosis is still dismal. Cognisant to this, it is necessary to identify more valuable biomarkers to enable effective $\mathrm{HBP}$ cancer prognosis and design of optimised treatment strategies.

Serum ferritin (SF) is a major iron-binding protein that plays a critical role in tumour angiogenesis, proliferation and immune regulation. ${ }^{8-10}$ Studies have found that iron may possess the ability to induce mutations, mediated through free radical generation or promote tumorigenesis through nutritional mechanisms. ${ }^{11} 12$ Iron accumulation in the liver is an auxiliary factor that causes liver injury and hepatocarcinogenesis. ${ }^{13}$ Elevated SF can reveal excessive iron load. High SF has been reported to be closely associated with inflammation, liver disease and cancer. ${ }^{810} 14-16$ Previous studies have demonstrated that high $\mathrm{SF}$ is associated with worse prognosis in lung cancer, colorectal cancer, PC and peripheral T-cell lymphoma. ${ }^{10}$ 17-19 However, the prognostic role of SF in HCC is controversial. ${ }^{20} 21$

To date, no meta-analysis has been conducted to assess the prognostic role of SF in HBP cancers. Herein, a meta-analysis was conducted to identify the prognostic role of pretreatment SF in HBP cancers. 


\section{MATERIALS AND METHODS}

\section{Search strategies}

A Preferred Reporting Items for Systematic Reviews and Meta-Analyses (PRISMA) statement-compliant systematic literature search for HBP cancer-related articles was conducted in the PubMed, EMBASE, Web of Science and Cochrane Library databases. The search was set to identify articles published from January 1970 to January 2020. The search terms used were: 'hepatocellular carcinoma', 'liver cancer', 'HCC', 'cholangiocarcinoma', 'biliary tract cancer', 'gallbladder cancer' and 'pancreatic cancer'. In the same line, free words adopted were: 'ferritin', 'isoferritin', 'prognosis', 'prognostic' and 'survival'. References from relevant articles were manually screened and retrieved for eligible studies. The PRISMA checklist is provided in online supplemental file 1 , and the complete search strategy for HBP cancer-related articles can be found in online supplemental file 2.

\section{Inclusion criteria}

A study was included for meta-analysis if: (1) diagnosis of HBP cancers had been confirmed based on clinicopathological data, (2) the study evaluated the relationship between pretreatment SF and OS, recurrence-free survival (RFS) / progression-free survival (PFS) or time to recurrence (TTR), and (3) the study presented the cutoff value of $\mathrm{SF}$.

\section{Exclusion criteria}

Studies were excluded if they were: (1) letters, case reports, reviews, abstracts, non-clinical studies or comments, (2) non-English articles, (3) studies presenting insufficient data for calculating the HR and 95\% CI, and (4) studies with duplicate data or repeat report.

\section{Data extraction and assessment of the risk of bias}

Two reviewers independently screened all included articles and extracted the relevant data. Any disagreement was resolved by consulting with a third reviewer. Data collected from each study included name of first author, year of publication, study region, type of publication, cancer type, number of patients involved, time of follow-up, treatment modality used, patient age, SF cutoff value, disease stage, survival analysis method (univariate or multivariate) and survival outcome (OS, PFS, RFS and TTR). HRs with their 95\% CIs were extracted from the univariate or multivariate survival analysis method or calculated from Kaplan-Meier survival curves. ${ }^{22}$

Quality evaluation of each included study was done using the Newcastle-Ottawa Scale (NOS). ${ }^{23}$ The NOS includes eight items categorised into three: case selection (0-4 points), group comparability ( $0-2$ points) and clinical outcome (0-3 points). Studies with a NOS score greater than or equal to 6 were defined as high-quality studies. $^{23}$

\section{Statistical analysis}

The meta-analysis was done using the STATA statistical software V.15.1 (STATA, College Station, Texas).

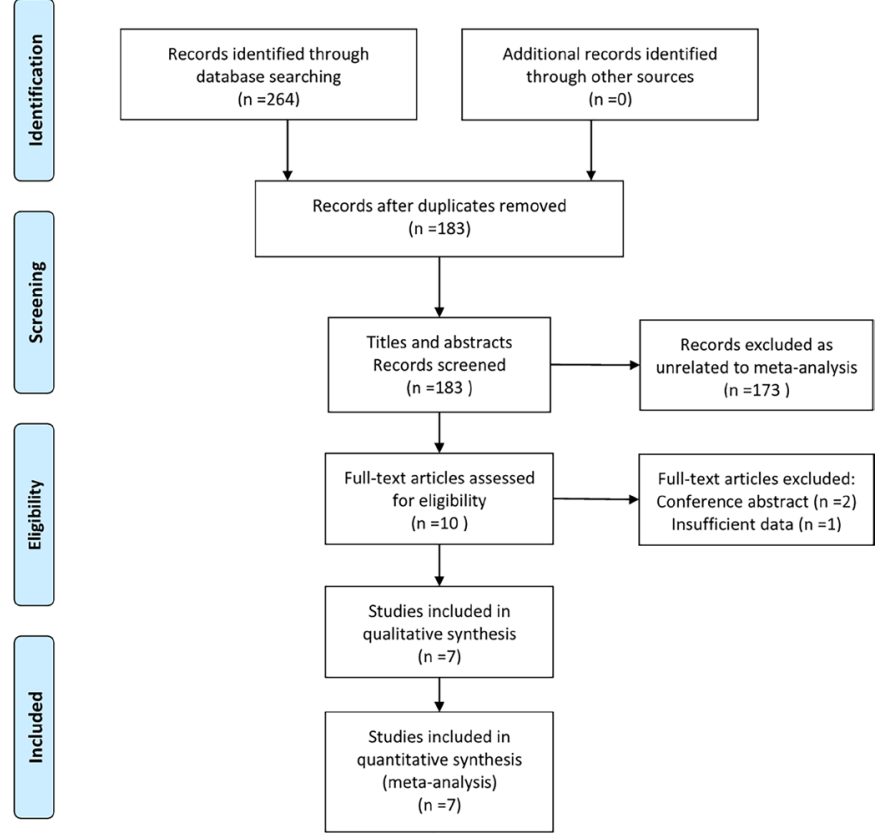

Figure 1 Flow chart of the included studies.

Cochran's $Q$ test and $\mathrm{I}^{2}$ test were employed to estimate the statistical heterogeneity between studies. ${ }^{24} \mathrm{~A} p$ value $<0.1$ for Cochran's $Q$ test or an $\mathrm{I}^{2}$ value more than $50 \%$ $(>50 \%)$ was defined as significant heterogeneity. ${ }^{25}$ In cases of significant heterogeneity, the random effects model was employed to calculate the pooled result. Otherwise, the fixed effects model was applied. HRs with 95\% CI were extracted from the included articles or calculated according to the methods reported by Tierney et al and Parmar et al. ${ }^{22} 26$ The HRs were then pooled to assess the prognostic outcome. Subgroup analyses were conducted based on the cancer type, race, SF cut-off value, tumournode-metastasis (TNM) stage and NOS score to detect the sources of heterogeneity. Sensitivity analyses were also undertaken to assess the stability of the pooled results. P values $<0.05$ indicated that there were statistically significant differences between groups.

\section{Patient and public involvement}

There was no patient or public involvement in the development of this research.

\section{RESULTS}

\section{Search results}

Search results and process are highlighted in figure 1. Two hundred and sixty-four relevant articles were identified from the database search. One hundred and eightythree articles remained after eliminating the duplicates (81 articles). The 183 articles were reviewed by checking their titles and abstracts for eligibility. Among them, 173 articles were removed because they were unrelated to the study subject. The 10 articles remaining underwent full-text screening. Three of them were excluded because they were conference abstract and thus had 
insufficient data. Finally, the seven studies remaining comprising 1244 patients were included in the metaanalysis. ${ }^{16}$ 19-21 27-29

\section{Characteristics of the included studies}

The full texts of the seven studies included for metaanalysis had been published between 2014 and 2019 . Most of the included studies were retrospective. Three studies were based in China, and the rest in Japan, Korea, USA and Italy. Three studies focused on HCC, two focused on PC while the other two focused on intrahepatic cholangiocarcinoma (ICC) and hepatobiliary cancer (HBC), respectively. The number of patients in each study ranged from 79 to 427 . All seven studies revealed the association between pretreatment $\mathrm{SF}$ and OS. Four studies also reported RFS/PFS/TTR data. PFS in one study had been calculated using the Kaplan-Meier method. ${ }^{19}$ The cut-off values of SF were between 200 and 840 . HRs with $95 \%$ CIs were directly obtained from the seven studies through univariate and/or multivariate analyses. The NOS scores of the included studies ranged from 5 to 9 . The specific features of the included studies are described in table 1.

\section{Meta-analysis}

SF and OS in HBP cancers

All studies explored the association between SF and OS in HBP cancers. Pooled results revealed that elevated SF was associated with poor OS (HR 1.60, 95\% CI 1.36 to 1.88 , $\mathrm{p}<0.001$ ) (figure 2). A fixed effects model was employed because there was no significant heterogeneity between the studies $\left(\mathrm{I}^{2}=37.1 \%, \mathrm{p}=0.146\right)$.

Subgroup analyses were conducted based on cancer type, ethnicity, TNM stage, cut-off value of SF and NOS score (table 2). High SF was associated with worse OS in patients with HBP cancers regardless of the cancer type (HCC, PC, ICC and HBC), race (Asian and Caucasian), SF cut-off value ( $\leq 250$ and $>250$ ), TNM stage (III-IV and I-IV) and NOS score $(\leq 7$ and $>7)$.

\section{SF and RFS/PFS/TTR in HBP cancers}

Four studies comprising 901 participants had data revealing the relationship between SF and RFS/PFS/TTR in HBP cancers. A random effects model was employed because of the significant heterogeneity between the studies $\left(p=0.003, I^{2}=78.0 \%\right)$. Pooled results revealed that elevated SF was associated with worse prognosis for RFS/PFS/TTR (HR $1.70,95 \%$ CI 1.15 to $2.52, \mathrm{p}=0.008$ ) (figure 3 ).

\section{Sensitivity analysis}

A sensitivity analysis was carried out to assess the effect of individual studies on the overall estimate (figure 4). The influence of the single data sets on the pooled HRs for OS was assessed by excluding one study at a time. No single study had a significant influence on the overall effect size. This indicated that the results were credible and stable.

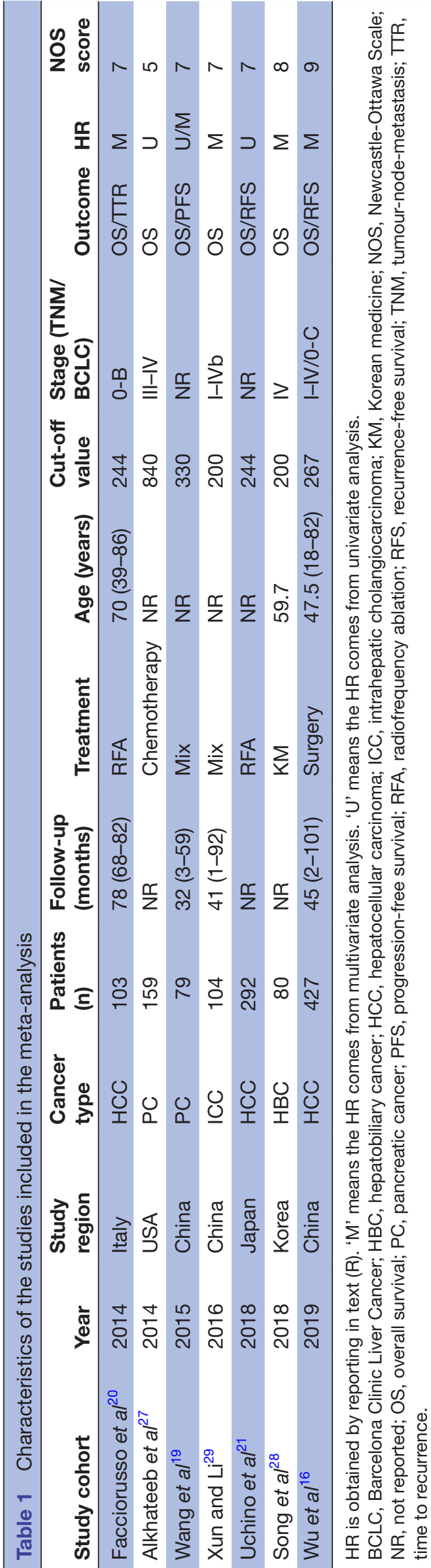




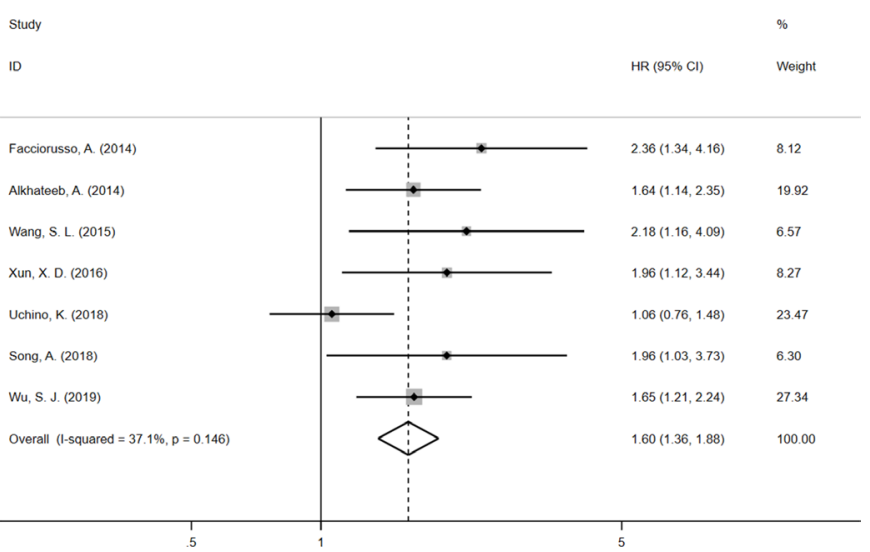

Figure 2 Meta-analysis of the association between pretreatment serum ferritin (SF) and overall survival (OS) of patients with hepatobiliary and pancreas (HBP) cancers.

Results are presented as individual and pooled HRs and 95\% Cls.

\section{DISCUSSION}

\section{The prognostic value of SF in HBP cancers}

In recent years, the number of patients diagnosed with HBP cancers has increased all over the world. ${ }^{125} 3031$ The majority of the patients diagnosed with HBP cancers are usually at an advanced stage. Despite the significant improvements made in the treatment of HBP cancers, their prognosis remains dismal. ${ }^{256}$ Precise prognostic forecast of patients with HBP cancers is critical for making

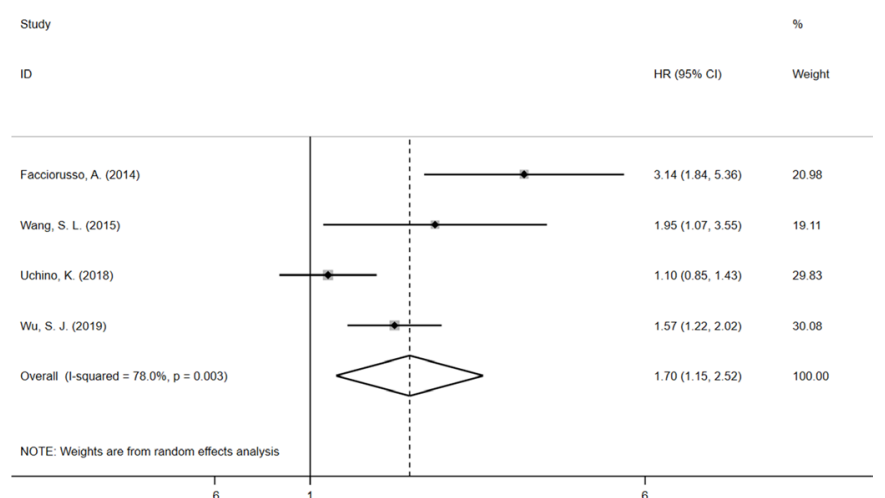

Figure 3 Meta-analysis of the association between serum ferritin (SF) and recurrence-free survival (RFS)/progressionfree survival (PFS)/time to recurrence (TTR) of hepatobiliary and pancreas (HBP) cancers. Results are presented as individual and pooled $\mathrm{HRs}$ and $95 \%$ Cls.

further management decisions. Inflammation can result in tumour initiation, progression and dissemination. It is further related to increasing epithelial to mesenchymal transition. ${ }^{32}$ Serum inflammatory indices, such as lymphocyte to monocyte ratio, ${ }^{33-35}$ platelet to lymphocyte ratio and neutrophil to lymphocyte ratio, ${ }^{36-39}$ have been found to have better prognostic value in patients with HBP cancer. Similarly, pretreatment SF is a novel inflammatory factor elevated in multiple human malignant tumours. It is associated with survival prognosis in

Table 2 Pooled HRs for OS according to subgroup analyses

\begin{tabular}{|c|c|c|c|c|c|c|c|}
\hline \multirow[b]{2}{*}{ Subgroup } & \multirow[b]{2}{*}{ Studies (n) } & \multirow[b]{2}{*}{ Patients (n) } & \multirow[b]{2}{*}{ Effects model } & \multirow[b]{2}{*}{ HR (95\% Cl) } & \multirow[b]{2}{*}{$P$ value } & \multicolumn{2}{|c|}{ Heterogeneity } \\
\hline & & & & & & $I^{2}(\%)$ & $\mathrm{Ph}$ \\
\hline Overall & 7 & 1244 & Fixed & 1.596 (1.358 to 1.876$)$ & $<0.001$ & 37.1 & 0.146 \\
\hline $\mathrm{HCC}$ & 3 & 633 & Random & 1.53 (1.01 to 2.32 ) & 0.043 & 70.9 & 0.032 \\
\hline $\mathrm{PC}$ & 2 & 238 & Fixed & 1.76 (1.29 to 2.41$)$ & $<0.001$ & 0 & 0.443 \\
\hline $\mathrm{HBC}$ & 1 & 80 & - & 1.96 (1.03 to 3.73$)$ & 0.04 & - & - \\
\hline \multicolumn{8}{|l|}{ Race } \\
\hline Asian & 5 & 982 & Fixed & 1.52 (1.25 to 1.83$)$ & $<0.001$ & 45.9 & 0.116 \\
\hline Caucasian & 2 & 262 & Fixed & 1.82 (1.34 to 2.43$)$ & $<0.001$ & 11.2 & 0.288 \\
\hline \multicolumn{8}{|l|}{ Cut-off value } \\
\hline \multicolumn{8}{|l|}{ TNM stage } \\
\hline III-IV & 2 & 239 & Fixed & 1.71 (1.25 to 2.35$)$ & 0.001 & 0 & 0.636 \\
\hline I-IV & 2 & 531 & Random & 1.71 (1.31 to 2.25$)$ & $<0.001$ & 0 & 0.591 \\
\hline \multicolumn{8}{|l|}{ NOS score } \\
\hline$\leq 7$ & 5 & 737 & Random & 1.67 (1.22 to 2.29$)$ & 0.001 & 55.6 & 0.061 \\
\hline$>7$ & 2 & 507 & Fixed & 1.70 (1.29 to 2.25$)$ & $<0.001$ & 0 & 0.632 \\
\hline
\end{tabular}

HBC, hepatobiliary cancer; HCC, hepatocellular carcinoma; ICC, intrahepatic cholangiocarcinoma; NOS, Newcastle-Ottawa Scale; OS, overall survival; PC, pancreatic cancer; $\mathrm{Ph}, \mathrm{p}$ values of $\mathrm{Q}$ test for heterogeneity test; TNM, tumour-node-metastasis. 


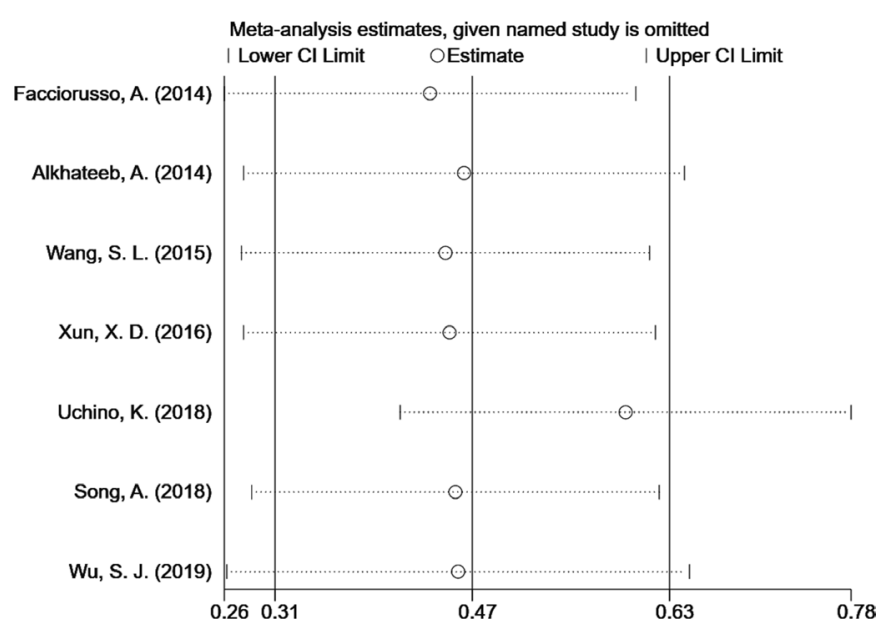

Figure 4 Chart of sensitivity analysis.

patients with HBP cancers. ${ }^{16} 192829$ Cognisant to this, SF may serve as a novel prognostic factor for patients with HBP cancer. To the authors' knowledge, this is the first meta-analysis that assesses the prognostic value of pretreatment SF in HBP cancers. So far, there is only one meta-analysis found that high SF may predict poor OS in patients with cancer (acute myeloid leukaemia and myelodysplastic syndromes). ${ }^{40}$

\section{High SF predicted worse prognosis in HBP cancers}

This meta-analysis combined seven studies comprising 1244 patients. Elevated pretreatment SF was associated with worse OS and RFS/PFS/TTR in patients with HBP cancers. Subgroup analysis further revealed that high SF was significantly associated with shorter OS in patients with $\mathrm{HBP}$ cancers regardless of cancer type, race, SF cut-off value, TNM stage and NOS score. Consequently, pretreatment SF may be considered as an available prognostic biomarker for patients with HBP cancers. These results were proved to be credible and stable based on sensitivity analysis. However, considering that the sample sizes included in this study are relatively limited, our results are in need of cautious interpretation. SF is a widely available, routinely measured and cost-effective inflammatory biomarker. Therefore, SF can be routinely tested as a clinical index before treatment in patients with HBP cancers. The levels of pretreatment SF might be used as a predictive tool for monitoring treatment response. Elevated pretreatment SF levels that indicated poor prognosis of patients with HBP cancers would help practitioners provide optimal care and management for these patients. Consequently, SF could be used as a practical reference for predicting the prognosis and adjusting the treatment strategy in patients with HBP cancers. Further studies ascertaining the biological basis of the association between pretreatment SF and survival outcome may result in uncovering new therapeutic targets for the development of innovative, biologically based adjuvant therapy for HBP cancers.
The underlying molecular mechanisms of SF related to HBP cancer prognosis

The specific molecular mechanisms involved in the prognostic effect of SF in HBP cancers remain unknown. SF plays a vital role in maintaining iron balance as one of the main storage and carrier proteins of iron. Iron plays a significant role in DNA synthesis and cell proliferation. ${ }^{41}$ Tumour cells need more iron than normal cells because of their differences in cell regenerative capacity. ${ }^{18}$ Besides storing iron and maintaining iron homeostasis, SF participates in immune regulation, tumour angiogenesis and proliferation..$^{8-10132042} \mathrm{SF}$ synthesis is induced by hepatocytes and macrophages. SF has been reported to be overexpressed in tumour-associated macrophages..$^{8-10} 184344 \mathrm{In}$ addition, SF can bind high molecular weight kininogen thereby inhibiting its antiangiogenic effect. ${ }^{45}$ It is also capable of inhibiting the proliferation of haematopoietic progenitor cells and lymphocytes. ${ }^{46}{ }^{47}$ Further to this, it may play an additional role in promoting tumour cell proliferation through a non-iron-mediated mechanism. ${ }^{43}$ To sum up, high SF levels may indicate a proinflammatory environment, antitumour immune activity and/or antitumour proliferation ability. It is thus not surprising that $\mathrm{SF}$ is associated with an unfavourable prognosis in patients with cancer which has again been confirmed by our meta-analysis.

\section{The limitation of this meta-analysis}

Nevertheless, this study was limited by several factors. Most of the included studies were retrospective that may have led to selection bias and thus affected the reliability of our results. However, interestingly, the sensitivity analysis indicated that our results were credible and stable. Even so, large-scale multicentre prospective cohorts are required to clarify the prognostic value of pretreatment SF in HBP cancers. In the same line, there were insufficient data to explore the relationship between pretreatment $\mathrm{SF}$ and clinicopathological features. Therefore, additional studies are needed to address this issue. Moreover, all the studies were published in English and thus could cause publication bias. In addition, publication bias may exist as most studies included in this meta-analysis had significant findings, which is due to the fact that journals are more likely to publish studies with significant findings. Finally, the cut-off value of SF varied among studies that might lead to heterogeneity between studies. In view of this, we conducted subgroup analyses on cut-off value of SF in this study. In the future, the optimal cut-off value of SF should be validated for further research needs and clinical application.

\section{CONCLUSION}

This study demonstrated that elevated pretreatment SF was associated with worse prognosis in patients with HBP cancers. As such, it may serve as a novel prognostic biomarker for HBP cancers. Future research which is well designed and large scale is required to clarify this conclusion. 
Acknowledgements We thank the authors of all the included studies.

Contributors SWL and CGJ conceived the study, and participated in the designing and drafting of the manuscript. SWL, YHF and YL retrieved the literature. YHF, YL, ZKM and XCH collected the data. CGJ and SWL participated in the design of the study and performed the statistical analysis. ZXJ revised the manuscript. All authors read and approved the final manuscript.

Funding This research was supported by grants from the Social Science and Technology Development Foundation of Dongguan, China (No 202050715025194), the National Natural Science Foundation of China (Grant No 81972792) and the National Science Foundation of Guangdong Province, People's Republic of China (Grant No 2020A1515010149).

Competing interests None declared.

Patient consent for publication Not required.

Ethics approval It was waived as this study was only based on the summary result of previously published articles and individual patient data were not obtained or accessed.

Provenance and peer review Not commissioned; externally peer reviewed.

Data availability statement Data are available upon reasonable request.

Supplemental material This content has been supplied by the author(s). It has not been vetted by BMJ Publishing Group Limited (BMJ) and may not have been peer-reviewed. Any opinions or recommendations discussed are solely those of the author(s) and are not endorsed by BMJ. BMJ disclaims all liability and responsibility arising from any reliance placed on the content. Where the content includes any translated material, BMJ does not warrant the accuracy and reliability of the translations (including but not limited to local regulations, clinical guidelines, terminology, drug names and drug dosages), and is not responsible for any error and/or omissions arising from translation and adaptation or otherwise.

Open access This is an open access article distributed in accordance with the Creative Commons Attribution Non Commercial (CC BY-NC 4.0) license, which permits others to distribute, remix, adapt, build upon this work non-commercially, and license their derivative works on different terms, provided the original work is properly cited, appropriate credit is given, any changes made indicated, and the use is non-commercial. See: http://creativecommons.org/licenses/by-nc/4.0/.

ORCID iD

Shuwen Lin http://orcid.org/0000-0002-0621-5067

\section{REFERENCES}

1 Bray F, Ferlay J, Soerjomataram I, et al. Global cancer statistics 2018: GLOBOCAN estimates of incidence and mortality worldwide for 36 cancers in 185 countries. CA Cancer J Clin 2018;68:394-424.

2 Augustine MM, Fong Y. Epidemiology and risk factors of biliary tract and primary liver tumors. Surg Oncol Clin N Am 2014;23:171-88.

3 Jarnagin WR, Ruo L, Little SA, et al. Patterns of initial disease recurrence after resection of gallbladder carcinoma and hilar cholangiocarcinoma: implications for adjuvant therapeutic strategies. Cancer 2003;98:1689-700.

4 Li C, Wen T-F, Yan L-N, et al. Postoperative neutrophil-to-lymphocyte ratio plus platelet-to-lymphocyte ratio predicts the outcomes of hepatocellular carcinoma. J Surg Res 2015;198:73-9.

5 Ryan DP, Hong TS, Bardeesy N. Pancreatic adenocarcinoma. N Engl J Med 2014;371:1039-49.

6 Siegel RL, Miller KD, Jemal A. Cancer statistics, 2020. CA Cancer J Clin 2020;70:7-30.

7 Tabrizian P, Jibara G, Hechtman JF, et al. Outcomes following resection of intrahepatic cholangiocarcinoma. HPB 2015;17:344-51.

8 Alkhateeb AA, Connor JR. The significance of ferritin in cancer: antioxidation, inflammation and tumorigenesis. Biochim Biophys Acta 2013;1836:245-54.

9 Oikonomou T, Goulis I, Soulaidopoulos S, et al. High serum ferritin is associated with worse outcome of patients with decompensated cirrhosis. Ann Gastroenterol 2017;30:217-24.

10 Tingting H, Di S, Xiaoping C, et al. High preoperative serum ferritin predicted poor prognosis in non-metastatic colorectal cancer. Saudi Med J 2017;38:268-75.

11 Genaro-Mattos TC, Maurício Ângelo Q, Rettori D, et al. Antioxidant activity of caffeic acid against iron-induced free radical generation--a chemical approach. PLoS One 2015;10:e0129963.

12 Jomova K, Valko M. Importance of iron chelation in free radicalinduced oxidative stress and human disease. Curr Pharm Des 2011;17:3460-73.
13 Lambrecht RW, Sterling RK, Naishadham D, et al. Iron levels in hepatocytes and portal tract cells predict progression and outcomes of patients with advanced chronic hepatitis C. Gastroenterology 2011;140:1490-500.

14 Kowdley KV, Belt P, Wilson LA, et al. Serum ferritin is an independent predictor of histologic severity and advanced fibrosis in patients with nonalcoholic fatty liver disease. Hepatology 2012;55:77-85.

15 Tran TN, Eubanks SK, Schaffer KJ, et al. Secretion of ferritin by rat hepatoma cells and its regulation by inflammatory cytokines and iron. Blood 1997:90:4979-86.

16 Wu S-J, Zhang Z-Z, Cheng N-S, et al. Preoperative serum ferritin is an independent prognostic factor for liver cancer after hepatectomy. Surg Oncol 2019;29:159-67.

$17 \mathrm{Ji} \mathrm{M}$, Li X-D, Shi H-B, et al. Clinical significance of serum ferritin in elderly patients with primary lung carcinoma. Tumour Biol 2014;35:10195-9.

18 Koyama S, Fujisawa S, Watanabe R, et al. Serum ferritin level is a prognostic marker in patients with peripheral T-cell lymphoma. Int $J$ Lab Hematol 2017;39:112-7.

19 Wang S-L, Cao S, Wu R, et al. Serum ferritin predicted prognosis in patients with locally advanced pancreatic cancer. Future Oncol 2015;11:2905-10.

20 Facciorusso A, Del Prete V, Antonino M, et al. Serum ferritin as a new prognostic factor in hepatocellular carcinoma patients treated with radiofrequency ablation. J Gastroenterol Hepatol 2014;29:1905-10.

21 Uchino K, Tateishi R, Nakagomi R, et al. Serum levels of ferritin do not affect the prognosis of patients with hepatocellular carcinoma undergoing radiofrequency ablation. PLoS One 2018;13:e0200943.

22 Parmar MK, Torri V, Stewart L. Extracting summary statistics to perform meta-analyses of the published literature for survival endpoints. Stat Med 1998;17:2815-34.

23 Stang A. Critical evaluation of the Newcastle-Ottawa scale for the assessment of the quality of nonrandomized studies in metaanalyses. Eur J Epidemiol 2010;25:603-5.

24 Higgins J, Thompson S, Deeks J, et al. Statistical heterogeneity in systematic reviews of clinical trials: a critical appraisal of guidelines and practice. J Health Serv Res Policy 2002;7:51-61.

25 Higgins JPT, Thompson SG. Quantifying heterogeneity in a metaanalysis. Stat Med 2002;21:1539-58.

26 Tierney JF, Stewart LA, Ghersi D, et al. Practical methods for incorporating summary time-to-event data into meta-analysis. Trials 2007;8:16.

27 Alkhateeb A, Zubritsky L, Kinsman B, et al. Elevation in multiple serum inflammatory biomarkers predicts survival of pancreatic cancer patients with inoperable disease. J Gastrointest Cancer 2014;45:161-7.

28 Song A, Eo W, Kim S, et al. Significance of serum ferritin as a prognostic factor in advanced hepatobiliary cancer patients treated with Korean medicine: a retrospective cohort study. BMC Complement Altern Med 2018;18:176.

29 Xun X-D, Li Q. Surgical treatment of intrahepatic cholangiocarcinoma: a retrospective study of 104 cases. Cancer Biol Med 2016;13:469-73.

30 Kai K, Miyoshi A, Kitahara K, et al. Analysis of extrahepatic multiple primary malignancies in patients with hepatocellular carcinoma according to viral infection status. Int J Hepatol 2012;2012:495950.

31 Murakami T, Nishikawa H, Koshikawa Y, et al. Double primary cancers: intrahepatic cholangiocarcinoma with myocardial metastases and lung squamous cell carcinoma. Intern Med 2012;51:2329-35.

32 Rhim AD, Mirek ET, Aiello NM, et al. EMT and dissemination precede pancreatic tumor formation. Cell 2012;148:349-61.

33 Stotz M, Szkandera J, Stojakovic T, et al. The lymphocyte to monocyte ratio in peripheral blood represents a novel prognostic marker in patients with pancreatic cancer. Clin Chem Lab Med 2015;53:499-506.

34 Li G-J, Xu H-W, Ji J-J, et al. Prognostic value of preoperative lymphocyte-to-monocyte ratio in pancreatic adenocarcinoma. Onco Targets Ther 2016;9:1085-92.

35 Wu S-J, Lin Y-X, Ye H, et al. Lymphocyte to monocyte ratio and prognostic nutritional index predict survival outcomes of hepatitis $B$ virus-associated hepatocellular carcinoma patients after curative hepatectomy. J Surg Oncol 2016;114:202-10.

36 Qi Q, Geng Y, Sun M, et al. Clinical implications of systemic inflammatory response markers as independent prognostic factors for advanced pancreatic cancer. Pancreatology 2015;15:145-50.

37 Mano Y, Shirabe K, Yamashita Y-I, et al. Preoperative neutrophilto-lymphocyte ratio is a predictor of survival after hepatectomy for hepatocellular carcinoma: a retrospective analysis. Ann Surg 2013;258:301-5. 
38 Lu S-D, Wang Y-Y, Peng N-F, et al. Preoperative ratio of neutrophils to lymphocytes predicts postresection survival in selected patients with early or intermediate stage hepatocellular carcinoma. Medicine 2016;95:e2722.

39 Ma W, Zhang P, Qi J, et al. Prognostic value of platelet to lymphocyte ratio in hepatocellular carcinoma: a meta-analysis. Sci Rep 2016;6:35378

40 Yang Y, Tang Z, An T, et al. The impact of iron chelation therapy on patients with lower/intermediate IPSS MDS and the prognostic role of elevated serum ferritin in patients with MDS and AML: a metaanalysis. Medicine 2019;98:e17406.

41 Le NTV, Richardson DR. The role of iron in cell cycle progression and the proliferation of neoplastic cells. Biochim Biophys Acta 2002;1603:31-46.

42 Lee S, Song A, Eo W. Serum ferritin as a prognostic biomarker for survival in relapsed or refractory metastatic colorectal cancer. J Cancer 2016;7:957-64.
43 Alkhateeb AA, Han B, Connor JR. Ferritin stimulates breast cancer cells through an iron-independent mechanism and is localized within tumor-associated macrophages. Breast Cancer Res Treat 2013;137:733-44.

44 Alkhateeb AA, Leitzel K, Ali SM, et al. Elevation in inflammatory serum biomarkers predicts response to trastuzumab-containing therapy. PLoS One 2012;7:e51379.

45 Coffman LG, Parsonage D, D'Agostino R, et al. Regulatory effects of ferritin on angiogenesis. Proc Natl Acad Sci U S A 2009;106:570-5.

46 Broxmeyer HE, Williams DE, Geissler K, et al. Suppressive effects in vivo of purified recombinant human $\mathrm{H}$-subunit (acidic) ferritin on murine myelopoiesis. Blood 1989;73:74-9.

47 Fargion S, Fracanzani AL, Brando B, et al. Specific binding sites for $\mathrm{H}$-ferritin on human lymphocytes: modulation during cellular proliferation and potential implication in cell growth control. Blood 1991;78:1056-61. 